\title{
Insights in silica toxicity from the characterisation of industrial materials and ex vivo samples
}

Francesco Di Benedetto $^{1}$, Elena Belluso ${ }^{2}$

${ }^{1}$ Dipartimento di Scienze della Terra, Università degli Studi di Firenze, Firenze, Italy

2 Dipartimento di Scienze della Terra \& "G. Scansetti” Centro Interdipartimentale per lo studio degli Asbesti e di altri Particolati Nocivi, Università degli Studi di Torino, Torino, Italy

The health concerns linked to the exposure to respirable crystalline silica (RCS) have been the subject of an extensive clinical, epidemiological and laboratory research. Exposurerelated pneumoconiosis, i.e. silicosis, but also self-immune pathologies and even lung cancer were correlated to the silica exposure in occupational settings. A peculiar aspect of the silica toxicity deals with the fact that the pathogenic power of the silica dusts has been found controversially variable in relation to the features of the exposure, and to the type of occupational setting.

Recently, studies were devoted to gain additional information on the physical and chemical environment of RCS in the settings were exposure can occur, as well as on the environment were RCS interacts with the biological tissue. This latter task has been addressed by the study of $e x$ vivo forensic samples.

The present communication aims to review the emerging settings of new exposures, with special reference to the artificial stone issue, the methods by which industrial and $e x$ vivo samples can be properly characterised, and the main results achieved. 